Thomas Beschorner

Alexander Brink

Bettina Hollstein

Marc C. Hübscher

OlafSchumann Hrsg.

Wirtschafts- und

Unternehmensethik 


\section{Wirtschafts- und Unternehmensethik}


Thomas Beschorner - Alexander Brink .

Bettina Hollstein - Marc C. Hübscher .

Olaf Schumann

(Hrsg.)

\section{Wirtschafts- und \\ Unternehmensethik}

算 SpringerVS 
Hrsg.

Thomas Beschorner

Universität St.Gallen

St.Gallen, Schweiz

Bettina Hollstein

Universität Erfurt

Max-Weber-Kolleg für kultur- und

sozialwissenschaftliche Studien

Erfurt, Deutschland

Olaf Schumann

Tübingen, Deutschland
Alexander Brink

Universität Bayreuth

Bayreuth, Deutschland

Marc C. Hübscher

Hamburg, Deutschland

ISBN 978-3-658-16204-7 ISBN 978-3-658-16205-4 (eBook)
https://doi.org/10.1007/978-3-658-16205-4

Die Deutsche Nationalbibliothek verzeichnet diese Publikation in der Deutschen Nationalbibliografie; detaillierte bibliografische Daten sind im Internet über http://dnb.d-nb.de abrufbar.

(C) Springer Fachmedien Wiesbaden GmbH, ein Teil von Springer Nature 2020

Das Werk einschließlich aller seiner Teile ist urheberrechtlich geschützt. Jede Verwertung, die nicht ausdrücklich vom Urheberrechtsgesetz zugelassen ist, bedarf der vorherigen Zustimmung des Verlags. Das gilt insbesondere für Vervielfältigungen, Bearbeitungen, Übersetzungen, Mikroverfilmungen und die Einspeicherung und Verarbeitung in elektronischen Systemen.

Die Wiedergabe von allgemein beschreibenden Bezeichnungen, Marken, Unternehmensnamen etc. in diesem Werk bedeutet nicht, dass diese frei durch jedermann benutzt werden dürfen. Die Berechtigung zur Benutzung unterliegt, auch ohne gesonderten Hinweis hierzu, den Regeln des Markenrechts. Die Rechte des jeweiligen Zeicheninhabers sind zu beachten.

Der Verlag, die Autoren und die Herausgeber gehen davon aus, dass die Angaben und Informationen in diesem Werk zum Zeitpunkt der Veröffentlichung vollständig und korrekt sind. Weder der Verlag, noch die Autoren oder die Herausgeber übernehmen, ausdrücklich oder implizit, Gewähr für den Inhalt des Werkes, etwaige Fehler oder Äußerungen. Der Verlag bleibt im Hinblick auf geografische Zuordnungen und Gebietsbezeichnungen in veröffentlichten Karten und Institutionsadressen neutral.

Springer VS ist ein Imprint der eingetragenen Gesellschaft Springer Fachmedien Wiesbaden GmbH und ist ein Teil von Springer Nature.

Die Anschrift der Gesellschaft ist: Abraham-Lincoln-Str. 46, 65189 Wiesbaden, Germany 


\section{Inhalt}

I GELEITWORT $\ldots \ldots \ldots \ldots \ldots \ldots \ldots \ldots \ldots \ldots \ldots \ldots \ldots \ldots \ldots \ldots \ldots$

\section{ALLGEMEINE EINLEITUNG}

Zur Einführung: Entstehungskontext und Grundidee der Zeitschrift FÜR WiRTSCHAFTS- UND UNTERNEHMENSETHIK $\ldots \ldots \ldots \ldots \ldots \ldots \ldots \ldots \ldots \ldots$ Marc C. Hübscher und Thomas Beschorner

\section{III ÖKONOMIE, KULTUR, ETHIK}

Einleitung zu Teil III: Öкоnомie - Kultur - Етнік $\ldots \ldots \ldots \ldots \ldots \ldots \ldots \ldots$ Thomas Beschorner und Marc C. Hübscher

\section{A ÖKONOMISCHE THEORIEN}

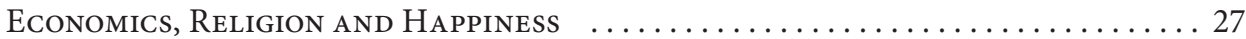

Lasse Steiner, Lisa Leinert and Bruno S. Frey

Der systematische Ort der Moral ist die Ethik! Einige kritische ANMERKungen zUR ÖKONOMischen ETHIK Karl Homanns $\ldots \ldots \ldots \ldots \ldots \ldots \ldots .45$ Michael S. Aßländer und Hans G. Nutzinger

Karl Homanns Programm einer ökonomischen Ethik. ,A View From Inside

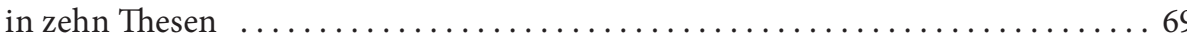

Korreferat zum Beitrag von Michael S. Aßländer und Hans G. Nutzinger Ingo Pies

Grenzmoral und Grenzen der Moral 83

Replik auf den Beitrag von Ingo Pies

Michael S. Aßländer und Hans G. Nutzinger 
Normetablierung, Normbefolgung, Normbestimmung. Beobachtungen und Bemerkungen zu Karl Homanns These „ÖKonomik - Fortsetzung

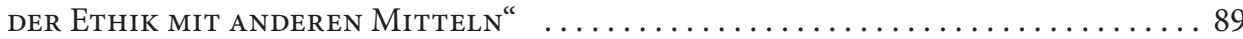
Eilert Herms

Ethik $=$ Ethik + Ökonomik $=$ Ökonomik ${ }^{2} \ldots \ldots \ldots \ldots \ldots \ldots \ldots \ldots \ldots \ldots \ldots$ Korreferat zum Beitrag von Eilert Herms

Michael Schramm

Rückblickendes Nachwort

Replik auf den Beitrag von Michael Schramm

Eilert Herms

INDIVIDUALETHIK ODER INSTITUTIONENETHIK: DiE RESOZIALISIERUNG

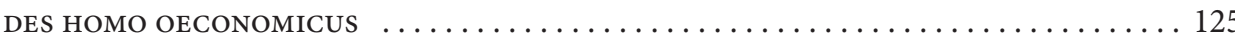

Peter Weise

Individualethik und Institutionenethik!

Korreferat zum Beitrag von Peter Weise

Matthias König

\section{B Kultur}

Wirtschaftsphilosophie - Die erweiterte Perspektive $\ldots \ldots \ldots \ldots \ldots \ldots \ldots 149$ Kurt Röttgers

Wirtschaftsphilosophie: Zur Neuorientierung einer Forschungsrichtung $\ldots \ldots 170$ Korreferat zum Beitrag von Kurt Röttgers

Olaf J. Schumann

Postscriptum

Kurt Röttgers

Kulturalistische Wirtschaftsethik. GrundzÜGe einer Theorie DER Anwendung 181

Thomas Beschorner

Desiderata einer kulturalistischen Wirtschaftsethik. Ein Kommentar zu Thomas Beschorners Forschungsprogramm 208

Korreferat zum Beitrag von Thomas Beschorner

Matthias Kettner 
Kulturalistische Wirtschaftsethik. Grundrisse eines (auch)

normativen Programms

Postscriptum

Thomas Beschorner

ETHICS IN THE ECONOMY

Viviana A. Zelizer

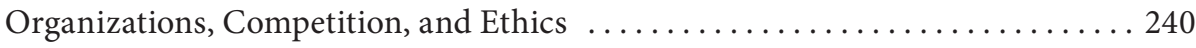

Commentary on the article by Viviana A. Zelizer

Diane Vaughan

Putting Codes in Perspective

Commentary on the article by Viviana A. Zelizer

Lynn S. Paine

Die Theorie des Humankapitals zwischen Kultur und ÖKonomie $\ldots . . . \ldots 249$ Dieter Thomä

Humankapital als Kulturalisierung der Ökonomie?

Korreferat zum Beitrag von Dieter Thomä

Matthias Jung

Frauen und Arbeit - Der Fähigkeitenansatz $\ldots \ldots \ldots \ldots \ldots \ldots \ldots \ldots \ldots$

Martha C. Nussbaum

Der Fähigkeitenansatz als Orientierung für eine feministische

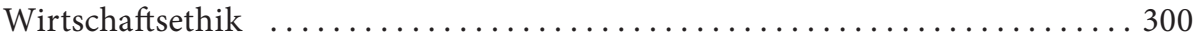

Korreferat zum Beitrag von Martha Nussbaum

Ulrike Knobloch

\section{Eтhiк}

NACHHALtigKeit - EIN KULTURELLES, BISHER ABER CHANCENLOSES

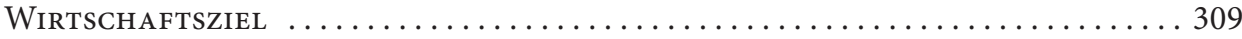

Klaus Michael Meyer-Abich

Pragmatische Physiozentrik oder die Wieder-entdeckung der Kultur 326

Korreferat zum Beitrag von Klaus Michael Meyer-Abich

Werner Theobald

WAS IST „GUTE“ SOZIOÖKONOMISCHE ENTWICKLUNG?

Eine wirtschaftsethische Perspektive

Peter Ulrich 
„Was ist ,gute` sozioökonomische Entwicklung?" aus philosophischer und entwicklungspolitischer Perspektive

Korreferat zum Beitrag von Peter Ulrich

Thomas Kesselring

Zur Kritik des entwicklungspolitischen Ökonomismus - zehn Jahre danach . . 358

Replik auf den Beitrag von Thomas Kesselring

Peter Ulrich

Corporate Human Rights Responsibilities

David Weissbrodt

Are "Human Rights" the "Business of Business"?

Commentary on the article by David Weissbrodt

Klaus M. Leisinger

Gibt es ein Menschenrecht auf Ethische Bildung?

389

Gerhard Kruip

Ethik oder Moral? Individuelle Verantwortung oder institutionelle

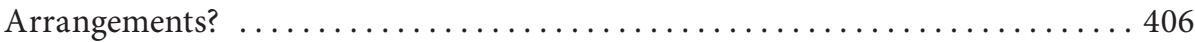

Korreferat zum Beitrag von Gerhard Kruip

Gertrud Nunner-Winkler

Wir brauchen jeweils beides: Ethik und Moral, individuelle Verantwortung und und moralförderliche Strukturen - eine Antwort auf Gertrud Nunner-Winkler .. 410 Replik auf den Beitrag von Getrud Nunner-Winkler

Gerhard Kruip

\section{WIRSCHAFTS- UND UNTERNEHMENSETHIK}

Einleitung ZU TeIl IV: Wirtschafts- Und Unternehmensethik $\ldots \ldots \ldots \ldots 415$ Alexander Brink und Olaf J. Schumann

\section{A Ordnungstheorie Und Ordnungsethik}

IDEEN DES GUTEN IN DER INTEGRATIVEN WIRTSCHAFTSETHIK.

HinfüHRUNG UND WeITERENTWICKLUNG $\ldots \ldots \ldots \ldots \ldots \ldots \ldots \ldots \ldots \ldots \ldots . \ldots \ldots$

Ulrike Knobloch

Unter welchen Bedingungen wäre eine Ethik des Guten eine gute Ethik? . . . . 452

Korreferat zum Beitrag von Ulrike Knobloch

Ingo Pies 
Gerechtigkeit in wirtschaftlicher Perspektive $\ldots \ldots \ldots \ldots \ldots \ldots \ldots \ldots 4$

Christofer Frey

Gerechtigkeit in der Ökonomik, von außen und innen betrachtet . . . . . . . 483

Korreferat zum Beitrag von Christofer Frey

Hans G. Nutzinger

Noch einmal: Das Thema der Gerechtigkeit im Blick auf die Ökonomie . . . . 491

Replik auf den Beitrag von Hans G. Nutzinger

Christofer Frey

Politische ÖKONOMie, Wirtschaftsethisch ReKonfiguriert.Funktionale SystemöKonomie im Kontext Praktischer Sozialökonomie . . . . . . . . . . . . 497 Peter Ulrich

Normativ vs. normal? Wider eine selbstgenügsame Wirtschaftsethik

in der akademischen Nische

Postscriptum

Peter Ulrich

European Economic Ethics Research. A Diagnosis $\ldots \ldots \ldots \ldots \ldots \ldots \ldots \ldots$

Adela Cortina

Vom Business Case zum Public Case? Der Beitrag privater

Selbstregulierung zu Global Governance $\ldots \ldots \ldots \ldots \ldots \ldots \ldots \ldots \ldots \ldots$

Klaus Dieter Wolf und Sandra Schwindenhammer

Vom Business Case zum Public Case! Frosch- und Vogelperspektiven

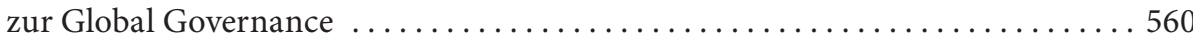

Korreferat zum Beitrag von Klaus Dieter Wolf und Sandra Schwindenhammer

Thomas Beschorner

Über die „Vogelperspektive“ als interdisziplinäre Klammer . . . . . . . . . . 563

Replik auf den Beitrag von Thomas Beschorner

Klaus Dieter Wolf und Sandra Schwindenhammer

Ist DIE ORDNUNGSÖKONOMIK ZUKUNFTSFÄHIG? . . . . . . . . . . . . . . . . . . . 569

Lars P. Feld und Ekkehard A. Köhler

Der normative, methodologische Individualismus.

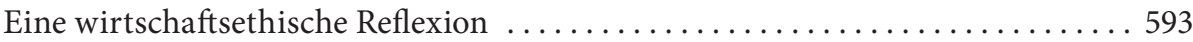

Korreferat zum Beitrag von Lars P. Feld und Ekkehard A. Köhler

Bettina Hollstein 


\section{B THEORIEN DER UNTERNEHMENSETHIK}

Business Ethics in the Intercultural and Global Context:

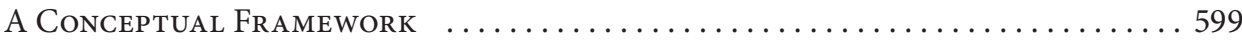

Georges Enderle

Probleme und Möglichkeiten einer globalen Wirtschaftsethik

Korreferat zum Beitrag von Georges Enderle

Hans G. Nutzinger

Building and Cultivating the Entire Field of Business and Economic Ethics . . . 622

Postscriptum

Georges Enderle

The Value-Added Approach to Business Ethics.

Ethical Reflections on the Challenges Facing International

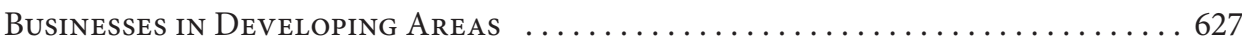

Frederick Bird

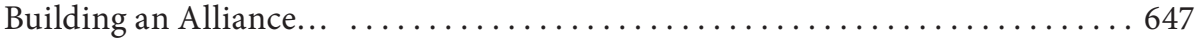

Commentary on the article by Frederick Bird

Hansjörg Elshorst

A Value-Added Approach to Business Ethics

Postscriptum

Frederick Bird

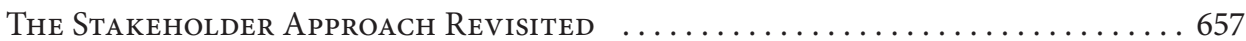

R. Edward Freeman

Stakeholder Theory between General and Contextual Approaches.

A German View ......................................... 672

Commentary on the article by R. Edward Freeman

Ursula Hansen, Matthias Bode und Dirk Moosmayer

Business Ethics in Germany. Problems, Concepts, and Functions $\ldots . \ldots .685$ Hans-Ulrich Küpper

Should Business be Moral? . . . . . . . . . . . . . . . . . . . . . . . . . . . . . . 706

Commentary on the article by Hans-Ulrich Küpper

Thomas Donaldson

Main Elements of Analytical Business Ethics

A Reply to Thomas Donaldson

Hans-Ulrich Küpper 


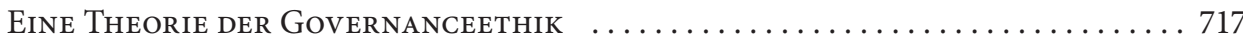
Josef Wieland

Governanceethik und philosophische Ethik mit ökonomischer Methode.

Versuch einer Verhältnisbestimmung . . . . . . . . . . . . . . . . . . . 740

Korreferat zum Beitrag von Josef Wieland

Karl Homann

\section{PROBLEMORIENTIERTE DISKURSE:}

\section{ANWENDUNGSBEREICHE UND THEORETISCHE IMPULSE}

Einleitung Zu Kapitel V: Problemorientierte Diskurse ANWENDUNGSBEREICHE UND THEORETISCHE IMPULSE $\ldots \ldots \ldots \ldots \ldots \ldots \ldots \ldots$ Bettina Hollstein und Thomas Hajduk

\section{A Praxis der Unternehmensethik}

Social Accountability 80oo. Ein Praktikables Instrument Zur IMPLEMENTIERUNG VON UNTERNEHMENSETHIK IN INTERNATIONAL TÄTIGEN UnTERNEHMEN? . . . . . . . . . . . . . . . . . . . . . . . . 777

Dirk Ulrich Gilbert

Der SA 8000 als ordnungspolitisches Instrument zur freiwilligen Umsetzung von Sozialstandards im Globalisierungsprozess $\ldots \ldots \ldots \ldots \ldots \ldots \ldots \ldots$. . . . . . 799 Korreferat zum Beitrag von Dirk Ulrich Gilbert Karl-Hermann Blickle

Social Accountability 8000. Ein deliberativer Ansatz zur Implementierung von Unternehmensethik in multinationalen Unternehmen? . . . . . . . . . . . . . . 804 Replik auf den Beitrag von Karl-Hermann Blickle Dirk Ulrich Gilbert

Environmental, Social and Governance Key Performance Indicators from a Capital Market Perspective 809 Alexander Bassen and Ana Maria Kovács

Intangibles Reporting - The Financial Communication Challenge in Response to Corporate Responsibility Requirements.

In Search for a Common Language

Commentary on the article by Alexander Bassen and Ana Maria Kovács

Dirk Schiereck and Anke Königs 
Unternehmensethik für Professional Service Firms.

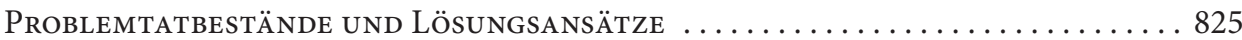

Andreas Georg Scherer und Jens Michael Alt

Professional Service Firms. Ein Härtetest für die Unternehmensethik! . . . . . 849

Korreferat zum Beitrag von Andreas Georg Scherer und Jens Michael Alt

Alfred Kieser

B Konsumethik PRivater Haushalte

Moral hybrids. SKizze ZU EINer Theorie MORAlischen Konsums

Birger P. Priddat

$a=a+b$

Korreferat zum Beitrag von Birger P. Priddat

Olaf J. Schumann

,Geliehene Moral ${ }^{\star}$

Postscriptum

Birger P. Priddat

Can The Socially Responsible Consumer Be Mainstream? 885

Timothy M. Devinney, Pat Auger and Giana Eckhardt

\section{EThik des Kapitalmarkts}

MORAL AN DIE BörsE?

Friedhelm Hengsbach SJ

„Dieses Mal ist alles anders“? Umsteuern zu einer regelethischen Reflexion . . . 911 Postscriptum

Friedhelm Hengsbach SJ

Renditedruck Der FinANZMÄrkTe - SCHWERE ZeIten FÜr DIE

UNTERNEHMENSETHIK

Bernhard Emunds

Institutionen- versus Individualethik? Eine falsche Alternative vor dem

Hintergrund der Finanzkrise

Korreferat zum Beitrag von Bernhard Emunds

Bettina Hollstein 
Weiter steigender oder wieder sinkender Renditedruck? . . . . . . . . . . . . 947

Replik auf den Beitrag von Bettina Hollstein

Bernhard Emunds

Zuviel Vertrauen? Über Moral und Finanzen $\ldots \ldots \ldots \ldots \ldots \ldots \ldots \ldots \ldots . \ldots 93$

Birger P. Priddat

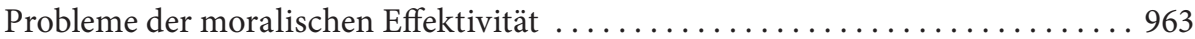

Postscriptum

Birger P. Priddat

\section{EThiK DES Dritten SeKtors}

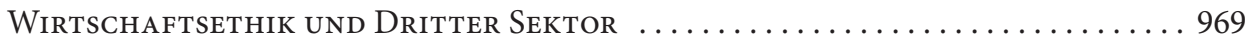
Karl Gabriel

Zivilgesellschaft: Dritter Sektor oder Dritter Weg?

Zur wirtschaftsethischen Bedeutung des Bürgerengagements $\ldots \ldots \ldots \ldots \ldots 96$

Korreferat zum Beitrag von Karl Gabriel

Peter Ulrich

Der systematische Ort der Zivilgesellschaft. Welche Rolle weist eine

ökonomische Theorie der Moral zivilgesellschaftlichen Organisationen

in der modernen Gesellschaft zu?

Korreferat zum Beitrag von Karl Gabriel

Ingo Pies und Stefan Hielscher

Corporate Governance in Non-Profit-Organisationen.

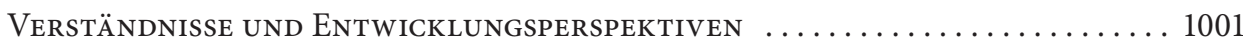

Michael Meyer und Florentine Maier

Warum Non-Profit-Organisationen in der $z f w u$ ? Non-Profit-Organisationen,

Corporate Governance und normatives Management ................ 1014

Korreferat zum Beitrag von Michael Meyer und Florentine Maier

Martin Büscher

Leadership in Sozialen Organisationen. Zur Organisation

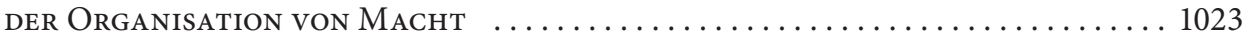

Arne Manzeschke und Eckhard Nagel

Leadership in sozialen Organisationen. Ergänzende Erwägungen

zum Untersuchungsobjekt . . . . . . . . . . . . . . . . . . . . . . . 1042

Korreferat zum Beitrag von Arne Manzeschke und Eckhard Nagel

Steffen Fleßa 


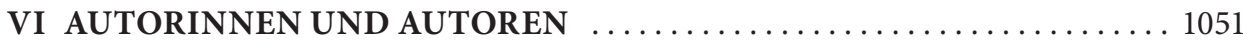


Geleitwort 
Als ich vor 20 Jahren in meiner Eigenschaft als Vorsitzender des Deutschen Netzwerks Wirtschaftsethik (DNWE) das Geleitwort zum ersten Heft der Zeitschrift für Wirtschaftsund Unternehmensethik ( $z f w u)$ verfasst habe, war ich beeindruckt von der Initiative und dem Wagemut der damaligen Herausgeber (Berliner Forum zur Wirtschafts- und Unternehmensethik). Zeitpunkt und Umstände schienen mir für die Gründung einer einschlägigen deutschen Zeitschrift günstig, gerade auch im Hinblick auf die ethischen Anforderungen an die Unternehmensführung im Zuge der Globalisierung. Der Erfolg hänge davon ab, so schrieb ich damals, dass es gelinge, das Organ für Theorie und Praxis gleichermaßen attraktiv zu machen.

Mit dem vorliegenden Aufsatzband wird nun gewissermaßen der Nachweis erbracht, dass das Unterfangen geglückt ist. Die Herausgeber haben die Hauptbeiträge aus den ersten 15 Jahrgängen der $z f w u$ für erneut publikationswürdig erachtet. Das zeigt ihr Vertrauen in die Qualität und Überlebenskraft der Zeitschrift; und dies in einer Situation, die durch einen verschärften globalen Wettbewerb unter den einschlägigen Zeitschriften gekennzeichnet ist, mit deutlichen Wettbewerbsvorteilen der (allseits bekannten) englischsprachigen Organe. Die Promotions- und Habilitationspolitik auch deutscher Universitäten (mit dem Trend zur kumulativen Qualifikation) trägt seit langem massiv dazu bei, dass die Kandidaten, bestärkt durch ihre akademischen Lehrer und zur Förderung der wissenschaftlichen Karriere, für ihre Aufsätze Publikationsorgane mit „höchster Reputation“ suchen, und diese sind - wenn man den Rankings glauben darf - auch im Bereich der Wirtschafts- und Unternehmensethik eher im englisch-sprachigen Kulturkreis angesiedelt. In deutscher Sprache verfasste Beiträge haben darüber hinaus sprachbedingt generell im globalen Kontext einen deutlich beschränkteren Absatzmarkt.

Diese strukturell bedingten Wettbewerbsnachteile werden durch die Politik der Herausgeber zu kompensieren versucht: die $z f w u$ wird über die Datenbanken von GENIOS (WISO), Proquest und EBSCO international verbreitet und Beiträge, die älter als ein Jahr sind, werden kostenfrei zum Abruf (Open Access) angeboten. Das hat in der Vergangenheit wohl wesentlich zur Festigung der Marktstellung der Zeitschrift beigetragen.

Auch der vorliegende Sammelband wird mit den abgedruckten Hauptbeiträgen ganz in diesem Sinne hilfreich sein. Er bringt nämlich insbesondere die komparativen Stärken 
der deutschen Diskussion in den ersten 15 Jahrgängen in der akademischen Szene (nochmals) zur Geltung. Diese Stärken liegen im Bereich der theoretischen (philosophischen, nationalökonomischen und betriebswirtschaftlichen) Fundierung der Wirtschafts- und Unternehmensethik und sind damit fast zwangsläufig stark interdisziplinär orientiert. Dabei versteht es sich aber von selbst, dass die internationale Theorieentwicklung durchaus berücksichtigt und verarbeitet wurde; nicht zuletzt die englischsprachigen Beiträge bekannter Verfasser aus verschiedenen Kulturkreisen in diesem Band legen davon Zeugnis ab. Zugleich wurde die Bedeutung der Praxis, wie einige Aufsätze zeigen, nicht vernachlässigt.

Alles in allem kann man feststellen, dass die $z f w u$ im deutschsprachigen Raum ohne Zweifel das führende Journal für wirtschafts- und unternehmensethische Fragestellungen ist. Da trifft es sich gut, dass in den letzten Jahren eine Reihe von grundlegenden Lehrbüchern zur Wirtschafts- und Unternehmensethik erschienen ist. Der vorgelegte Band ergänzt nämlich diese Lehrbuchliteratur durch seine problembezogene Facettenvielfalt und eignet sich deshalb durchaus als ergänzende Lektüre für einschlägige Lehrveranstaltungen. Man greift sicherlich nicht zu hoch, wenn man den vorgelegten Sammelband als einen wichtigen Beitrag zur Diskussion um die Wirtschafts- und Unternehmensethik bezeichnet, eine Diskussion, die in den letzten Jahren gerade auch durch viele jüngere Autoren einen beachtlichen Aufschwung genommen hat.

Nürnberg, den 30. März 2020

Horst Steinmann 